\title{
Evaluation of hematological parameters in dyspepsia patients infected with Helicobacter pylori
}

\author{
A retrospective study from the Central Region of Saudi Arabia
}

\author{
Ahmad A. AlShomar, MD.
}

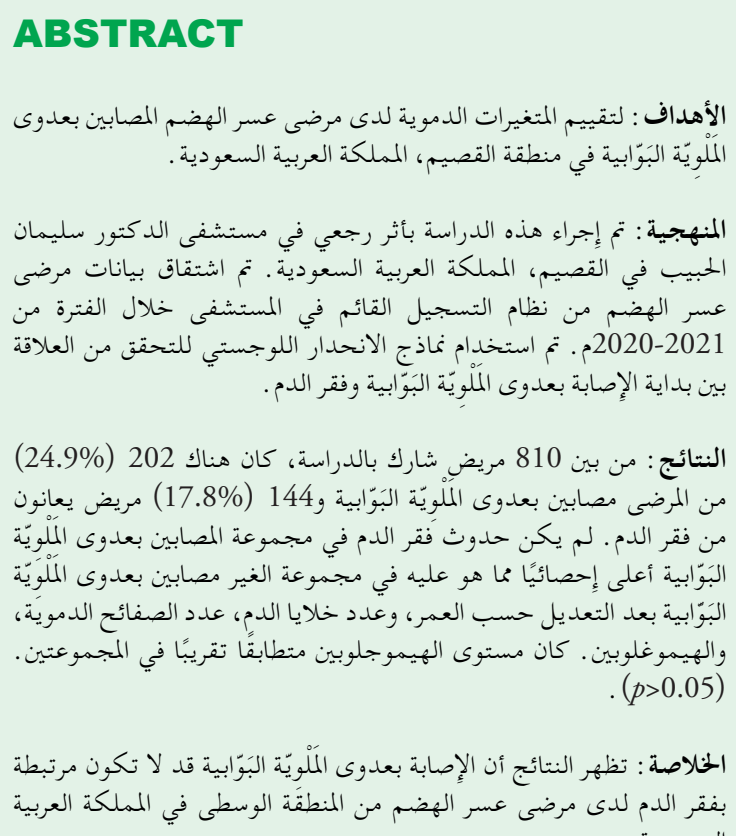

السعودية.

Objectives: To evaluate the hematological parameters in dyspepsia patients infected with Helicobacter pylori ( $H$. pylori) infection from Al Qassim province of Kingdom of Saudi Arabia (KSA).

Methods: This retrospective study was carried out in Dr. Sulaiman Al-Habib Hospital, Al Qassim, KSA. The data of dyspepsia and epigastric pain patients were derived from the hospital based registration system between 2020-2021. Logistic regression models were used to investigate the correlation between the onset of $H$. pylori infection and anemia.

Results: Among 810 enrolled patients, there were $202(24.9 \%)$ patients with $H$. pylori infection and $144(17.8 \%)$ with anemia. The prevalence of anemia in the $H$. pylori (+) group was not statistically higher than the $H$. pylori (-) group after adjusting age, red blood cell count, serum ferritin, mean corpuscular volume, platelet count, and mean corpuscular hemoglobin $(p>0.05)$. The level of hemoglobin was almost identical in both the $H$. pylori (+) group and the H. pylori (-) group $(p>0.05)$.

Conclusion: The findings show that $H$. pylori infection may not be related to anemia in dyspepsia patients from the Central region of KSA.

Keywords: hematology, anemia, dyspepsia, Helicobacter pylori, Saudi Arabia

Saudi Med J 2022; Vol. 43 (1): 75-80 doi: 10.15537/smj.2022.43.1.20210733

From the Department of Medicine, College of Medicine, Qassim University, and from Dr. Sulaiman Al Habib Medical Group, Buraidah, Kingdom of Saudi Arabia.

Received 12th September 2021. Accepted 23rd November 2021.

Address correspondence and reprint request to: Dr. Ahmad A. AlShomar, Department of Medicine, College of Medicine, Qassim University, Buraidah, Kingdom of Saudi Arabia.E-mail: A.alshomar@qu.edu.sa ORCID ID: https://orcid.org/0000-0002-5083-2732

$\mathrm{H}$ elicobacter pylori $(H$. pylori) infection is one of the most common chronic bacteria that infect humans. Overall, approximately half of the world's population is seropositive; yet, in some developing countries, the prevalence can reach as high as $80 \%$, while in developed countries, $40 \%$ of the population is infected. ${ }^{1}$ Helicobacter pylori is a gram-negative bacteria with a spiral shape that helps to colonize the human stomach effectively. ${ }^{2}$ Helicobacter pylori infections are directly associated with both benign and malignant gastric disorders. However, there are several extragastric manifestations involving a variety of medical subspecialties closely related to $H$. pylori infection. Hence, the involvement of $H$. pylori infection in many extra-gastric disorders remains an area of active 
research. ${ }^{3}$ Hematological consequences of $H$. pylori infection includes: iron deficiency anemia, vitamin B12 deficiency, idiopathic thrombocytopenic purpura, and gastric extranodal marginal zone lymphoma. ${ }^{4}$

Anemia is a significant public health problem across the world. It has been attributed to increased morbidity and mortality in women, child's development delay, cognitive impairment, increased risk of infection, and reduced productivity due to declining work capacity, resulting in a significant economic burden on the family and entire population. ${ }^{5}$ Established mechanisms for anemia includes: underproduction anemia, increased destruction, and blood loss. Once anemia has been diagnosed, a search for the underlying cause is necessary. The most common cause of anemia is an iron deficiency affecting over one billion people, majorly women, and children. ${ }^{6}$ Helicobacter pylori has been implicated as a possible etiology for anemia. Several epidemiological and clinical trials demonstrated a correlation between $H$. pylori infection and anemia. Nonetheless, conflicts emerge among the findings of several trials concerning the role of $H$. pylori in inducing anemia. ${ }^{7}$ The pathophysiologic processes by which $H$. pylori contributes to iron deficient anemia are not fully understood. It has been proposed that $H$. pylori infection stimulates the liver to produce a hormone called hepcidin. Hepcidin controls iron metabolism in enterocytes and releases stored iron from macrophages of the reticuloendothelial system, resulting in the condition termed as "anemia of inflammation". 8 Preliminary studies indicated that serum hepcidin levels were increased in patients with $H$. pylori infection and these levels returned to normal when the infection is eradicated. ${ }^{9}$ Chronic gastritis induced bleeding, which occurs in patients infected with $H$. pylori, is another possible mechanism of iron deficiency anemia. The prevalence of anemia and $H$. pylori infection are common in developing countries. However, the related available data of Saudi Arabia's population is still limited. ${ }^{10}$ Therefore, this study aimed at investigating the correlation between $H$. pylori infection and anemia among Saudi patients with unexplained anemia and to study the relationship between $H$. pylori infection and the hematological parameters of these patients through a case-control study.

Methods. A retrospective study was carried out on 810 Saudi patients (381 males and 429 females). The

Disclosure. Authors have no conflict of interests, and the work was not supported or funded by any drug company. inclusion criteria were as follow: Saudi patients who were tested for $H$. pylori infection using the $H$. pylori stool antigen test. Patients with dyspepsia and epigastric pain attending the inpatient and outpatient setting of Dr. Sulaiman Al-Habib Hospital in Al Qassim, Saudi Arabia, were enrolled between March 2020-2021. There were no gender, age, or body mass index limitations. The exclusion criteria was patients having a history of blood loss, such as gastrointestinal bleeding, were excluded as potential causes of anemia. Also, patients with incomplete hematological tests (hemoglobin and mean cell volume $[\mathrm{MCV}]$ ) were excluded from the study.

The ethical approval was obtained from the Research Ethics Committee of $\mathrm{Al} \mathrm{Habib} \mathrm{Research} \mathrm{Centre,}$ Al Qassim, Saudi Arabia (study no. RC 20.04.133). Written informed consents were obtain from all participated patients. All procedures carried out in this study involving the participants were in accordance with the Ethical Standards of the Institutional or National Research Committee and with the 1964 Helsinki Declaration and its later amendments or comparable Ethical Standards.

A medical record review of all recruited participants was carried out, and data related to demographic characteristics and the results of hematological parameters (hemoglobin, red blood cells [RBCs], platelets, $\mathrm{MCV}$, and mean corpuscular hemoglobin $[\mathrm{MCH}])$ were collected.

According to the World Health Organization (WHO) gender-based criteria, defined anemia as a hemoglobin level $<13 \mathrm{~g} / \mathrm{dL}$ for men and $<12 \mathrm{~g} / \mathrm{dL}$ for women. Iron-deficiency anemia is defined as serum ferritin level $<21 \mathrm{ng} / \mathrm{ml}$ and the hemoglobin level is less than adjusted values for age and gender. Serum ferritin was measured using the ARCHITECT $7 \mathrm{k} 59$ assay, a 2-step chemiluminescent microparticle immunoassay (CMIA). The correlation between anemia and $H$. pylori infection was studied.

Patients' stools were collected and examined for $H$. pylori antigen with an onsite $H$. Pylori Ag rapid test (CTK Biotech, Inc., USA, 2017).

Statistical analysis. The Prism-version 5 software was used for the data analysis, and the data was analyzed using ANOVA. Some data was also analyzed and validated by Statistical Package for Social Sciences, version 20 (IBM Corp., Armonk, NY, USA) using the Chi-square test. A $p$-value of $<0.05$ was considered significant.

Results. A total of 810 dyspepsia patients (mean age of $37.68 \pm 18.7$ years) were enrolled in this study, 
including 429 (53\%) females and 381 (47\%) males, who were suspected of $H$. pylori infection. Helicobacter pylori infection was assessed using the H. Pylori stool antigen test. The proportion of patients with $H$. pylori $(+)$ infection is $24.9 \%$, while patients without H. pylori (-) infection is $75.1 \%$. There was no significant difference between $H$. pylori status and age $(p=0.388)$, hemoglobin $(p=0.289), \mathrm{RBC}$ count $(p=0.292)$, serum ferritin $(p=0.775), \operatorname{MCV}(p=0.358)$, platelet $(p=0.948)$, and $\mathrm{MCH}(p=0.296)$. The complete data is summarized in Table 1 and the gender based distribution among the studied patients with and without $H$. pylori infection is presented in Figure 1.

Patients were also subdivided into 2 groups according to $H$. pylori antigen status; a group of $H$. pylori $\mathrm{Ag}$ positive $(\mathrm{n}=202)$ and a group of $H$. pylori $\mathrm{Ag}$ negative ( $\mathrm{n}=608)$. The group of $H$. pylori positive was further divided into 2 groups; group anemic $(\mathrm{n}=35)$ and non-anemic $(\mathrm{n}=167)$. There was a significant difference in hemoglobin level, RBC, MCV, MCH, and serum ferritin between anemic and non-anemic patients infected with $H$. pylori $(p<0.05)$.

Anemia was found in 35/202 (17.3\%) patients infected by $H$. pylori and $99 / 608$ (16.3\%) patients without $H$. pylori infection. The complete results are summarized in Table 2.

Figure 2 demonstrates the prevalence pattern of anemic patients infected with or without $H$. pylori. The data pointed out that the difference between the 2 groups was not statistically significant $(p>0.05)$.

Discussion. This study aims at determining the occurrence of anemia in dyspepsia patients suspected of $H$. pylori infection in Al Qassim province of Saudi Arabia. A total of 810 patients were studied first to check the prevalence of $H$. pylori infection.

Table 1 - Hematological characteristics in H. pylori positive and negative patients.

\begin{tabular}{lccc}
\hline Parameters & \multicolumn{2}{c}{ H. pylori infection } & $P$-value \\
& Positive $(\mathbf{n}=202)$ & Negative $(\mathbf{n}=608)$ & \\
\hline Male/female (ratio) & Mean $\pm S D$ & \\
Age (years) & M=103/F=99 (1.04) & M=278/F=330 (0.84) & - \\
Hemoglobin & $38.66 \pm 1.177$ & $37.35 \pm 0.7851$ & 0.3879 \\
RBC & $13.83 \pm 0.1376$ & $13.67 \pm 0.07388$ & 0.2889 \\
Serum ferritin & $4.962 \pm 0.04242$ & $4.913 \pm 0.02315$ & 0.2916 \\
MCV & $58.71 \pm 3.622$ & $57.61 \pm 1.854$ & 0.7748 \\
Platelet & $86.31 \pm 0.4327$ & $86.77 \pm 0.2483$ & 0.3576 \\
MCH & $275.7 \pm 5.018$ & $276.1 \pm 3.227$ & 0.9483 \\
\hline
\end{tabular}

H. pylori: Helicobacter pylori, RBC: red blood cell count, MCV: mean corpuscular volume, $\mathrm{MCH}$ : mean corpuscular hemoglobin, SD: standard deviation

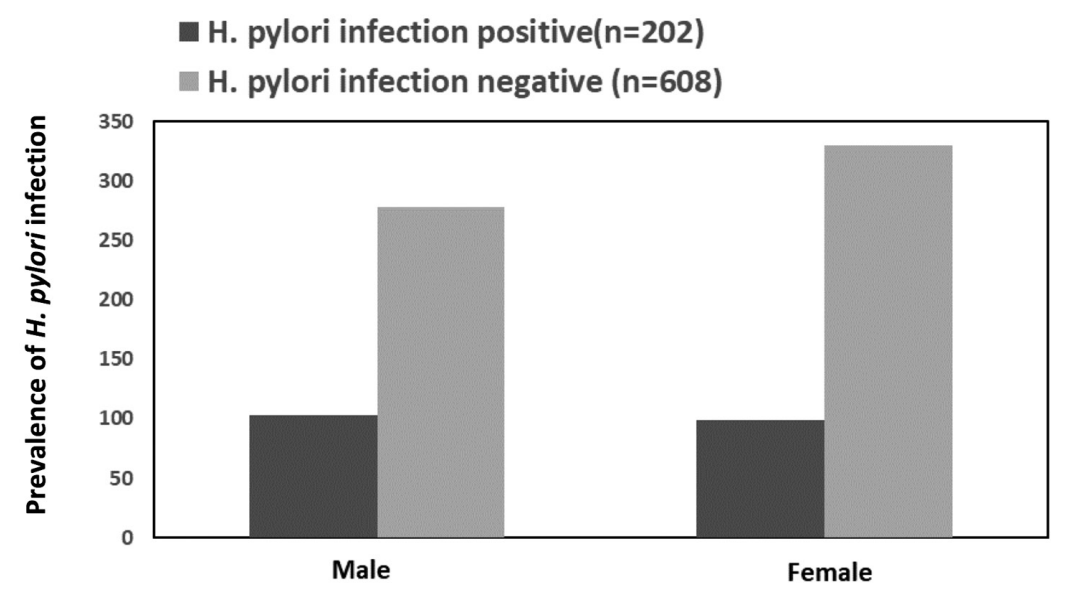

Figure 1 - Gender wise distribution of patients with and without Helicobacter pylori (H. pylori) infection. 
Table 2 - Hematological characteristics in anemic and non-anemic patients infected with or without H. pylori infection.

\begin{tabular}{|c|c|c|c|c|c|c|}
\hline \multirow[t]{3}{*}{ Parameters } & \multicolumn{3}{|c|}{ H. pylori infection positive $(\mathrm{n}=202)$} & \multicolumn{3}{|c|}{ H. pylori infection negative $(\mathrm{n}=608)$} \\
\hline & $\mathrm{Hb}<12 \mathrm{gm} / \mathrm{dl}(\mathrm{n}=35)$ & $\mathrm{Hb} \geq 12 \mathrm{gm} / \mathrm{dl}(\mathrm{n}=167)$ & $P$-value & $\mathrm{Hb}<12 \mathrm{gm} / \mathrm{dl}(\mathrm{n}=99)$ & $\mathrm{Hb} \geq 12 \mathrm{gm} / \mathrm{dl}(\mathrm{n}=509)$ & $P$-value \\
\hline & \multicolumn{3}{|c|}{ Mean \pm SD } & \multicolumn{2}{|c|}{ Mean \pm SD } & \\
\hline Male/female (ratio) & $\mathrm{M}=5 / \mathrm{F}=30(0.167)$ & $\mathrm{M}=98 / \mathrm{F}=69(1.420)$ & - & $\mathrm{M}=13 / \mathrm{F}=86(0.151)$ & $\mathrm{M}=265 / \mathrm{F}=244(1.09)$ & - \\
\hline Prevalence (\%) & 17.3 & 82.7 & 0.0001 & 16.3 & 83.7 & 0.0001 \\
\hline Age(years) & $41.83 \pm 3.29$ & $38.00 \pm 1.24$ & 0.2190 & $36.56 \pm 2.01$ & $37.50 \pm 0.85$ & 0.6563 \\
\hline Hemoglobin & $10.77 \pm 0.15$ & $14.47 \pm 0.11$ & 0.0001 & $10.90 \pm 0.10$ & $14.21 \pm 0.062$ & 0.0001 \\
\hline $\mathrm{RBC}$ & $4.39 \pm 0.10$ & $5.08 \pm 0.04$ & 0.0001 & $4.40 \pm 0.05$ & $5.01 \pm 0.02$ & 0.0001 \\
\hline $\mathrm{MCV}$ & $80.02 \pm 1.43$ & $87.62 \pm 0.35$ & 0.0001 & $80.81 \pm 0.73$ & $87.92 \pm 0.23$ & 0.0001 \\
\hline Serum ferritin & $13.06 \pm 4.97$ & $68.27 \pm 3.87$ & 0.0001 & $14.08 \pm 1.65$ & $66.08 \pm 1.98$ & 0.0001 \\
\hline Platelet & $277.8 \pm 5.4$ & $274.3 \pm 5.071$ & 0.6407 & $307.7 \pm 10.98$ & $269.9 \pm 3.14$ & 0.0001 \\
\hline $\mathrm{MCH}$ & $24.51 \pm 0.57$ & $28.51 \pm 0.38$ & 0.0001 & $24.82 \pm 0.29$ & $30.28 \pm 1.02$ & 0.0192 \\
\hline
\end{tabular}

H. pylori: Helicobacter pylori, Hb: Hemoglobin, RBC: red blood cell count, MCV: mean corpuscular volume, MCH: mean corpuscular hemoglobin, SD: standard deviation

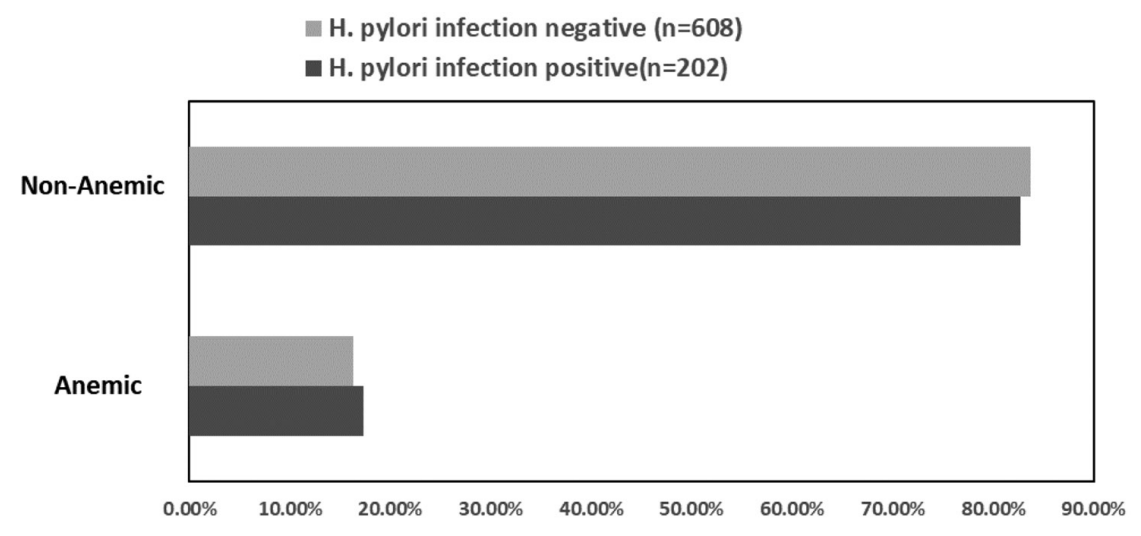

Figure 2 - Prevalence pattern of anemia in patients infected with or without Helicobacter pylori (H. pylori).

Approximately $24.9 \%$ of them were found to have H. pylori (+) infection. The prevalence of $H$. pylori positivity varies from region to region. In 2017, Hooi et al, ${ }^{11}$ carried out a systematic review and meta-analysis on the worldwide prevalence of $H$. pylori infection. They pointed out a wide variation in the prevalence of $H$. pylori infection between regions and countries. They reported that the prevalence was highest in Africa followed by Latin America, the Caribbean, and Asia. However, the rate of $H$. pylori infection was lowest in Northern America and Oceania. ${ }^{11}$ In highly developed countries, such as the Western World, the prevalence of $H$. pylori infection has decreased during the previous 2 decades, while the prevalence has risen in developing and newly industrialized countries. These variations in the prevalence of $H$. pylori infection are reflected in the variation of the socioeconomic status of the nations at the level of urbanization, sanitation, and the availability to access hygienic food and clean water. ${ }^{11}$ The variation in the prevalence of $H$. pylori infection was not only reported among different countries but these variations have also been reported significantly even within the same country. ${ }^{12}$ Importantly, the variation in the prevalence of $H$. pylori infection has been reported in different regions of Saudi Arabia. ${ }^{13,14}$ Few studies on $H$. pylori in the country have shown a high prevalence in various age groups of patients. ${ }^{13}$ Recently, variation in $H$. pylori infection among Saudi children was also seen in metropolitan cities such as Jeddah and Riyadh. ${ }^{13,15}$ It is also important to point out that the variation in the prevalence of $H$. pylori infection from region to region has important implications on the global future prevalence of sequelae associated with $H$. pylori, as this infection has been found to be associated with other chronic disorders including gastric cancer and disorders associated with anemia. ${ }^{16,17}$ The current study aims at analyzing the association of $H$. pylori infection with age, hemoglobin, RBC count, serum levels of ferritin, 
MCV, platelet count, and MCH. In order to determine these factors, the levels of these factors were analyzed in patients with confirmed $H$. pylori $(+)$ cases and the levels were compared with $H$. pylori (-) cases. The data point out no significant association of $H$. pylori infection with the patients' age, hemoglobin levels, RBC count, serum ferritin, $\mathrm{MCV}$, and platelet count with $\mathrm{MCH}$ levels $(p>0.05)$. These findings have been supported by a number of previously studied reports carried out among different populations. ${ }^{18,19}$ However, several findings were also reported. ${ }^{20,21}$ These variations may be due to the differences in the social and economic status of different populations.

Anemia has now been considered as a major public health issue worldwide and its prevalence is often associated with numerous disorders including: chronic disorders associated with kidney dysfunctions, malaria, schistosomiasis, and so on. ${ }^{22}$ Several studies explored an association of $H$. pylori infection with anemia in different regions of the world, but controversies are all around. ${ }^{7}$ Some of them considered that anemia is a complication of $H$. pylori infection, whereas others have reported no correlation between them. ${ }^{7}$ In order to determine an association of $H$. pylori infection with anemia in Saudi patients, they were subdivided into 2 groups according to $H$. pylori antigen status, namely, a group of $H$. pylori $(+)$ and a group of $H$. pylori $(+)$. The group of $H$. pylori $(+)$ was further divided into 2 groups; group anemic and nonanemic. The data showed a significant difference in hemoglobin level, RBC, $\mathrm{MCV}, \mathrm{MCH}$, and serum ferritin between anemic and non-anemic patients infected with $H$. pylori $(p<0.05)$. Moreover, the data also determined that anemia was found in $17.3 \% H$. pylori $(+)$ cases and $16.3 \%$ anemia in $H$. pylori (-) cases. In general, the data showed no association of anemia with $H$. pylori infection. These findings were consistent with a number of studies. ${ }^{23}$ However, different observations were also reported by others as well. ${ }^{24,25}$ This may be due to the variations in the characteristics of the patients from different populations. The underlying reasons for these variations among different populations still requires further research. The implications of the findings in this study provided an understanding of the relationship between anemia and patients infected with $H$. pylori. These findings support the controversy of previous studies regarding the role of $H$. pylori in causing anemia.

Study limitations. Despite its attempt to study the correlation between $H$. pylori infection and anemia in the Saudi population, this study has not confirmed the actual causality between $H$. pylori infection and anemia, as the study was an observational study, which might not be the optimal design for establishing a causal association between $H$. pylori infection and anemia. Besides, the study was carried out on dyspeptic patients who might have many underlying conditions as confounding factors that could not be fully controlled, impacting the statistical correlations between $H$. pylori infection and anemia. In addition, the study has been carried out only on patients from a single hospital (Dr. Sulaiman Al-Habib Hospital, Al Qassim, Saudi Arabia), which has made it impossible to generalize the findings to all of Saudi Arabia.

In conclusion, this study has indicated that $H$. pylori infection may not be related to anemia in dyspepsia patients.

Acknowledgment. The author gratefully acknowledge Dr. Sulaiman Al Habib Medical Group's Research Centre, Saudi Arabia, for their tremendous support, and the participation of all involved patients is also gratefully acknowledged. I would also like to thank RASAYELY (https://www.rasayely.com) for English language editing.

\section{References}

1. Mezmale L, Coelho LG, Bordin D, Leja M. Review: epidemiology of Helicobacter pylori. Helicobacter 2020; 25: e12734.

2. Denic M, Touati E, De Reuse H. Review: pathogenesis of Helicobacter pylori infection. Helicobacter 2020; 25: e12736.

3. Pellicano R, Ianiro G, Fagoonee S, Settanni CR, Gasbarrini A. Review: extragastric diseases and Helicobacter pylori. Helicobacter 2020; 25: e12741.

4. Santambrogio E, Orsucci L. Helicobacter pylori and hematological disorders. Minerva Gastroenterol Dietol 2019; 65: 204-213.

5. Janbek J, Sarki M, Specht IO, Heitmann BL. A systematic literature review of the relation between iron status/anemia in pregnancy and offspring neurodevelopment. Eur J Clin Nutr 2019; 73: 1561-1578.

6. James SL, Abate D, Abate KH, Abay SM, Abbafati C, Abbasi $\mathrm{N}$, et al. Global, regional, and national incidence, prevalence, and years lived with disability for 354 diseases and injuries for 195 countries and territories, 1990-2017: a systematic analysis for the Global Burden of Disease Study 2017. Lancet 2018; 392: 1789-1858.

7. Hudak L, Jaraisy A, Haj S, Muhsen K. An updated systematic review and meta-analysis on the association between Helicobacter pylori infection and iron deficiency anemia. Helicobacter 2017; 22.

8. Mendoza E, Duque X, Hernández Franco JI, Reyes Maldonado E, Morán S, Martínez G, et al. Association between active H. pylori infection and iron deficiency assessed by serum hepcidin levels in school-age children. Nutrients 2019; 11: 2141.

9. Lee SY, Song EY, Yun YM, Yoon SY, Cho YH, Kim SY, et al. Serum prohepcidin levels in Helicobacter pylori infected patients with iron deficiency anemia. Korean J Intern Med 2010; 25: 195-200. 
10. Nasif WA, Ali ASE, Alamodi HS, Alrefai AA, Alzubedi AA, Almasoudi HS, et al. Impact of Helicobacter pylori on hematological parameters among Saudi population. Saudi Med J 2021; 42: 643-648.

11. Hooi JKY, Lai WY, Ng WK, Suen MMY, Underwood FE, Tanyingoh D, et al. Global prevalence of Helicobacter pylori infection: systematic review and meta-analysis. Gastroenterology 2017; 153: 420-429.

12. Lin Y, Kawai S, Sasakabe T, Nagata C, Naito M, Tanaka K, et al. Effects of Helicobacter pylori eradication on gastric cancer incidence in the Japanese population: a systematic evidence review. Jpn J Clin Oncol 2021; 51: 1158-1170.

13. Akeel M, Elmakki E, Shehata A, Elhafey A, Aboshouk T, Ageely $\mathrm{H}$, et al. Prevalence and factors associated with $H$. pylori infection in Saudi patients with dyspepsia. Electron Physician 2018; 10: 7279-7286.

14. Al-Hussaini AA, Al Jurayyan AN, Bashir SM, Alshahrani D. Where are we today with Helicobacter pylori infection among healthy children in Saudi Arabia? Saudi J Gastroenterol 2019; 25: 309-318.

15. Hasosah M, Satti M, Shehzad A, Alsahafi A, Sukkar G, Alzaben A, et al. Prevalence and risk factors of Helicobacter pylori infection in Saudi children: a three-year prospective controlled study. Helicobacter 2015; 20: 56-63.

16. Tsay FW, Hsu PI. H. pylori infection and extra-gastroduodenal diseases. J Biomed Sci 2018; 25: 65.

17. Franceschi F, Zuccalà G, Roccarina D, Gasbarrini A. Clinical effects of Helicobacter pylori outside the stomach. Nat Rev Gastroenterol Hepatol 2014; 11: 234-242.
18. Saler T, Keşkek ŞÖ, Kırk S, Ahbab S, Ortoğlu G. H. pylori may not be associated with iron deficiency anemia in patients with normal gastrointestinal tract endoscopy results. Adv Hematol 2014; 2014: 375915.

19. John S, Baltodano JD, Mehta N, Mark K, Murthy U. Unexplained iron deficiency anemia: does Helicobacter pylori have a role to play? Gastroenterol Rep (Oxf) 2018; 6: 215-220.

20. Xu MY, Cao B, Yuan BS, Yin J, Liu L, Lu QB. Association of anaemia with Helicobacter pylori infection: a retrospective study. Sci Rep 2017; 7: 13434.

21. Hou B, Zhang M, Liu M, Dai W, Lin Y, Li Y, et al. Association of active Helicobacter pylori infection and anemia in elderly males. BMC Infect Dis 2019; 19: 228.

22. Chaparro CM, Suchdev PS. Anemia epidemiology, pathophysiology, and etiology in low- and middle-income countries. Ann N Y Acad Sci 2019; 1450: 15-31.

23. Santos IS, Minten GC, Valle NC, Tuerlinckx GC, Boccio J, Barrado DA, et al. Helicobacter pylori and anemia: a communitybased cross-sectional study among adults in Southern Brazil. Cad Saude Publica 2009; 25: 2653-2660.

24. Rahat A, Kamani L. Frequency of iron deficiency anemia (IDA) among patients with Helicobacter pylori infection. PakJ Med Sci 2021; 37: 776-781.

25. Demerdash DME, Ibrahim H, Hassan DM, Moustafa H, Tawfik NM. Helicobacter pylori associated to unexplained or refractory iron deficiency anemia: an Egyptian single-center experience. Hematol Transfus Cell Ther 2018; 40: 219-225. 